Claremont Colleges

Scholarship@ Claremont

Pomona Faculty Publications and Research

Pomona Faculty Scholarship

$12-2-1996$

\title{
Magnetic Levitation and Noncoalescence of Liquid Helium
}

\author{
M.A. Weilert \\ Dwight L. Whitaker \\ Pomona College \\ H. J. Maris \\ G. M. Seidel
}

\section{Recommended Citation}

"Magnetic Levitation and Noncoalescence of Liquid Helium" M. A. Weilert, D. L. Whitaker, H. J. Maris, and G. M. Seidel, Phys Rev. Lett, 77, 4840 (1996).

This Article is brought to you for free and open access by the Pomona Faculty Scholarship at Scholarship @ Claremont. It has been accepted for inclusion in Pomona Faculty Publications and Research by an authorized administrator of Scholarship @ Claremont. For more information, please contact scholarship@cuc.claremont.edu. 


\title{
Magnetic Levitation and Noncoalescence of Liquid Helium
}

\author{
M. A. Weilert, D. L. Whitaker, H. J. Maris, and G. M. Seidel \\ Department of Physics, Brown University, Providence, Rhode Island 02912
}

(Received 20 August 1996)

\begin{abstract}
We describe experiments in which drops of liquid helium-4, as large as $2 \mathrm{~cm}$ in diameter, are magnetically levitated. We have found that, when two or more drops are levitated in the same magnetic trap, the drops often remain in a state of apparent contact without coalescing. It appears that this effect is caused by the slow evaporation of liquid from the drops. [S0031-9007(96)01730-9]

PACS numbers: 85.25.Ly, 47.55.Dz, 67.90.+z, 75.20.Ck
\end{abstract}

The possibility of suspension of objects without material support has been of interest for centuries. In the laboratory a number of different levitation techniques have been developed [1]. Objects have been levitated through the action of acoustic or electromagnetic radiation, and levitation can also be achieved through the use of suitable static electric or magnetic fields. Here we report the magnetic levitation of large drops of liquid helium, in both the superfluid and normal states. In these experiments we have observed a surprising phenomenon: Levitated liquid drops can, under certain circumstances, come into contact but fail to coalesce.

A material of magnetic susceptibility $\chi$ placed in a static magnetic field experiences a force per unit volume of $\chi \nabla B^{2} / 2$. Since helium is diamagnetic, it is repelled from a region of high field. In order to levitate an object, the magnitude of $|B(d B / d z)|$ must exceed $\rho g /|\chi|$, where $\rho$ is the density of the material. Using the known susceptibility of helium, it then follows that the required value of $|B(d B / d z)|$ is $20.7 \mathrm{~T}^{2} \mathrm{~cm}^{-1}$ [2]. A variety of diamagnetic materials, both solid and liquid, have been levitated using room temperature Bitter magnets [3]. These magnets, which can provide a value of $|B(d B / d z)|$ sufficient to levitate helium, are, however, difficult to use for cryogenic applications due to vibration and the difficulties associated with having to work within a small bore that is at room temperature. Liquid hydrogen has been levitated using superconducting solenoids [4] which eliminate the difficulties associated with Bitter magnet solenoids. However, hydrogen only requires a value of $|B(d B / d z)|=5 \mathrm{~T}^{2} \mathrm{~cm}^{-1}$ to cancel the forces of gravity on the drop. Recently, improvements in the performance of magnet wire have made it possible to build a superconducting magnet that can provide the value of $|B(d B / d z)|$ required to levitate helium. We have used a superconducting solenoid especially designed for this purpose in which the inner coils are shorter than the outer windings. The inner coils of the magnet are made of $\mathrm{Nb}_{3} \mathrm{Sn}$ wire and the outer windings are $\mathrm{NbTi}$. The overall height of the solenoid is $14 \mathrm{~cm}$ and the bore is $3.2 \mathrm{~cm}$. The largest value of $|B(d B / d z)|$ that is achievable with this magnet is $22.5 \mathrm{~T}^{2} \mathrm{~cm}^{-1}$ which is obtained with a coil current of $118.7 \mathrm{~A}$ and an operating temperature below $2.2 \mathrm{~K}$.
To achieve stable levitation it is also necessary to have lateral stability. For this the magnitude of the magnetic field must increase for any horizontal displacement away from the levitation point. Thus, if the stable levitation point is to lie on the axis of the solenoid, the field must increase with increasing distance $r$ from the axis. From Maxwell's equations it is straightforward to show that this condition will be satisfied provided $(\partial B / \partial z)^{2}>$ $2 B\left(\partial^{2} B / \partial z^{2}\right)$. Thus, the stability requirement can be expressed entirely in terms of the field along the axis.

The potential energy per unit volume of helium in the magnetic field is

$$
U(r, z)=-\frac{\chi}{2} B^{2}(r, z)+\rho g z .
$$

The calculated contours of this potential energy function at a current of $118 \mathrm{~A}$ are shown in Fig. 1.

To introduce helium into the magnetic trap we have used a number of different techniques. It was possible to form a helium "mist" in the experimental cell, either

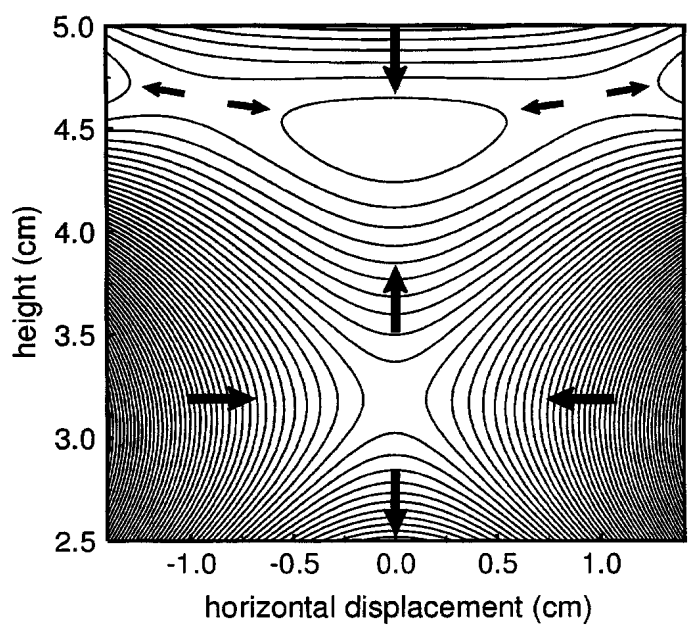

FIG. 1. Contours of potential energy per unit volume for liquid helium as a function of height from the center of the magnet coils and distance from the axis. The arrows point to regions of lower potential energy. Adjacent contours are displaced by $0.7 \mathrm{erg} \mathrm{cm}^{-3}$. The top of the innermost coil is located at a height of $z=4 \mathrm{~cm}$. 
by pumping on liquid contained in the lower part of the cell so as to make it boil violently or by rapid cooling of the experimental cell. Droplets in the mist agglomerate to form a large drop within the trap. Another method was to introduce liquid directly from a capillary which ended at the edge of the trap. This technique had the advantage that it was possible to introduce more than one drop into the trap. We were able to maintain drops in the trap indefinitely. The largest drops that could be levitated had a diameter of approximately $2 \mathrm{~cm}$.

The shape of a liquid-vapor interface in such a trap is determined by Laplace's equation $\kappa \sigma=P_{\text {liq }}$, where $\kappa$ is the curvature of the surface, $\sigma$ is the surface tension, $P_{\text {liq }}$ is the pressure of the liquid, and the pressure of the gas has, for the moment, been ignored. It follows that the height $z$ of the surface at radius $r$ satisfies the relation

$$
\frac{z_{r}+z_{r}^{3}+r z_{r r}}{r\left(1+z_{r}^{2}\right)^{3 / 2}} \sigma=\frac{\chi}{2} B^{2}(r, z)-\rho g z+C,
$$

where $C$ is a constant and $z_{r}$ and $z_{r r}$ are the first and second derivatives of $z$ with respect to $r$. This equation gives the surface shape for levitated drops and also describes the distortion of the surface of bulk liquid in the cell due to the magnetic potential. In the present apparatus we are able to view the drops only from above, i.e., looking down along the axis of the magnet. Thus we cannot determine the shape of a levitated drop in order to compare it to theory. However, Eq. (2) does make definite predictions for how the form of the surface should change as the cell is filled with liquid from the bottom. When the cell was filled from the bottom, a column of liquid rose up from the bottom of the cell, where the liquid was in contact with the cell walls. Upon filling the cell further, the top of the tower would expand radially and come into contact with the cell walls. This would result in an annular bubble located at the potential maximum on the cell wall seen in Fig. 1 at a height of $3 \mathrm{~cm}$. These observations are qualitatively consistent with predictions based on Eq. (2).

When two drops were introduced into the trap, it was frequently observed that the drops would come into contact with each other at the potential minimum, but would not coalesce. Drops were even seen to bounce off one another before coming into steady contact. Noncoalescence was observed between two drops with approximately the same size and between small and large drops. Sometimes groups of three or more drops were observed in apparent contact. For a current of $118 \mathrm{~A}$, two drops in contact were positioned side by side, i.e., with their centers at approximately the same height. This is to be expected given the potential energy contours shown in Fig. 1. At lower magnet currents the drops stacked one above the other, and this could be understood in terms of the change in shape of the potential energy contours for this current. Drops were observed in contact for as

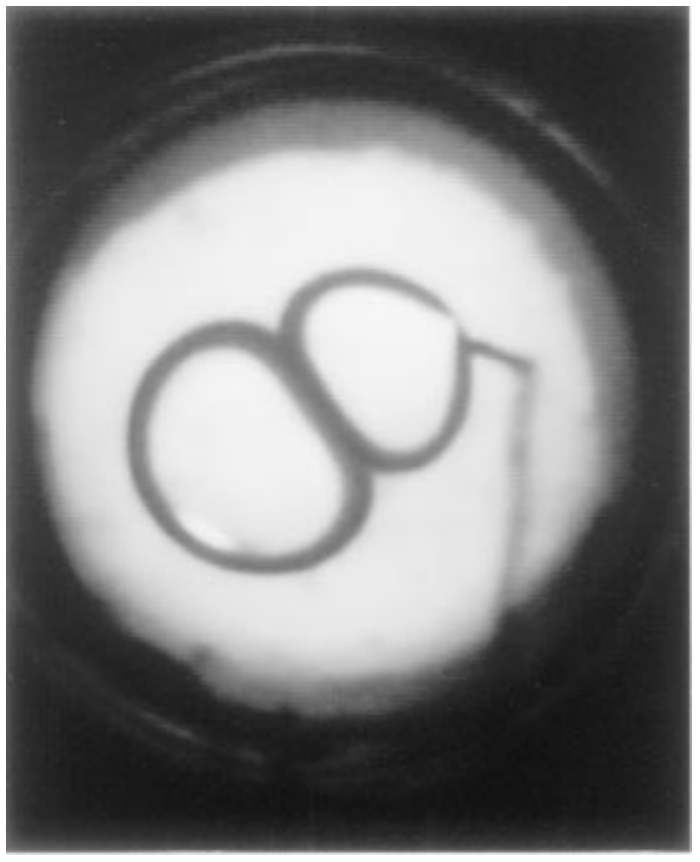

FIG. 2. Photograph of two drops in contact. The drops are viewed from above. The capillary which produced the two drops can be seen in contact with the drop on the right.

long as 3 min before coalescence occurred. Figure 2 is a photograph of two drops in contact.

As far as we could tell from our observations, two drops of equal size made contact over an area that was planar. The shape of the drops was similar to that of a liquid drop resting on a flat surface that the liquid does not wet. Thus the contact angle between the drop surface and the plane of contact was $180^{\circ}$. This implies that the total surface energy per unit area within the contact plane was nearly the same as the energy of two free drop surfaces.

We believe that the noncoalescence results from a layer of vapor between the drops which keeps the liquid surfaces from making direct contact with each other. But, it appears that this vapor layer is maintained because nonequilibrium conditions in the cell result in slow evaporation from each drop. The phenomenon is similar to the Leidenfrost effect [5] which is seen when water is dropped onto a hot metal surface, or when liquid nitrogen is spilled on the laboratory floor. Related observations on organic liquids have been made by Derjaguin and Prokhorov [6].

If an isolated spherical drop is placed in the cell and the temperature lowered, evaporation will take place at an equal rate everywhere on the surface of the drop. If another drop is nearby the evaporated vapor will have to escape from the region directly between the drops, and there will be a build up of pressure $\Delta P$ in this region. The two drops will approach each other until either contact is made or the pressure rise is sufficient to prevent further approach [Fig. 3(a)]. Assuming that the liquid and gas 


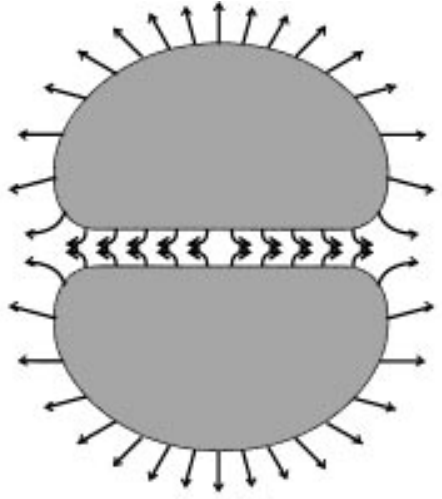

(a)

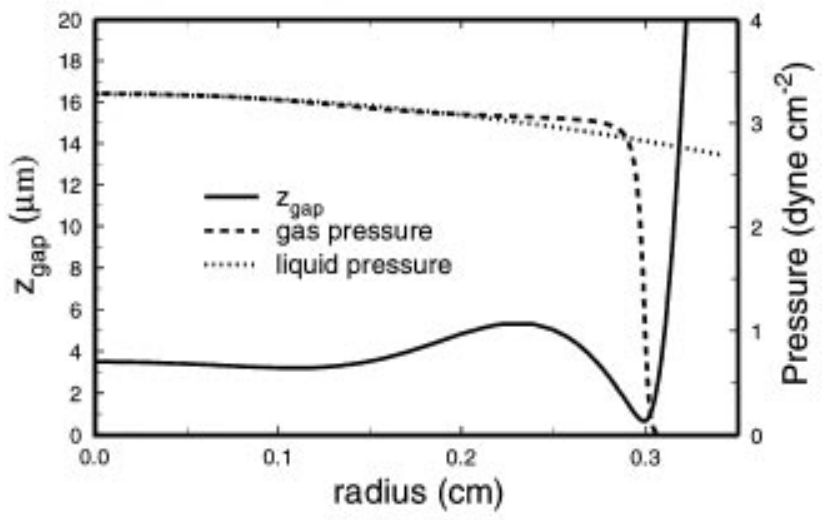

(b)

FIG. 3. (a) Schematic diagram of two drops which are prevented from coalescing by the pressure build up of evaporated vapor. The size of the gap between the drops is exaggerated. (b) Calculated shape of the drops in the region over the part of their surfaces which are nearly in contact. The solid line shows the distance to either drop from the symmetry plane of the gap as a function of radius from the center of the gap. The dotted and dashed lines show the pressures in the liquid and the gas, respectively, within the gap, relative to the ambient gas pressure. Note that there is a large difference between the scales for $z$ and $r$ axes.

are in equilibrium at the drop surface, there must be a temperature rise $\Delta T=\Delta P /(d P / d T)_{\mathrm{SVP}}$. For drops of a few $\mathrm{mm}$ radius the pressure that is required to balance the force exerted by the trap is only a few dyne $\mathrm{cm}^{-2}$. At $2.5 \mathrm{~K}$, for example, this corresponds to a temperature change $\Delta T$ of the order of only $10 \mu \mathrm{K}$.

Two observations support this interpretation: First, noncoalescence is seen only when the temperature of the cell is drifting down. Second, noncoalescence was never seen below the lambda point. As just mentioned, a pressure gradient in the gas requires a nonuniform temperature distribution within the drop which cannot exist in the superfluid phase.

We have carried out detailed calculations of the gas flow in the space between the drops and here briefly summarize the results. For simplicity we consider two drops of equal size whose centers lie along the $z$ axis. We first note that the required temperature variation $(10 \mu \mathrm{K})$ is very small compared to the temperature gradients likely to exist within the volume of a typical drop. If, for example, at $2.5 \mathrm{~K}$ a drop of radius $3 \mathrm{~mm}$ is in the cell and the cell pressure is decreasing at a rate of 1 torr $\min ^{-1}$ (a typical experimental value), the center of the drop will be $2.7 \mathrm{mK}$ hotter than the surface. Thus, the $10 \mu \mathrm{K}$ variation in surface temperature causes only a small variation in radial temperature gradient at the drop surface. It follows that the mass of liquid evaporated per unit area $\dot{J}$ will be almost uniform over the surface of the drop. Given $\dot{J}$, we can calculate the variation of the gap $2 z$ between the drops as a function of the distance $r$ from the center of plane of contact. Assuming that the gas flow is viscous and laminar, the pressure in the gas $P_{\text {gas }}$ must vary with $r$ according to

$$
\frac{d P_{\mathrm{gas}}}{d r}=-\frac{2 \pi r^{2} \dot{J}}{\rho_{\mathrm{gas}}} \frac{3 \eta_{\mathrm{gas}}}{8 \pi r z^{3}},
$$

where $\rho_{\text {gas }}$ and $\eta_{\text {gas }}$ are the gas density and viscosity, respectively. The curvature $\kappa$ of the liquid surface is related to the liquid and gas pressures by

$$
\kappa \sigma=P_{\text {liq }}-P_{\text {gas }} .
$$

For an assumed value of $\dot{J}$ corresponding to a particular rate of change of temperature or pressure in the cell, and for a given drop volume, these equations can be integrated to find the width of the gap between the drops as a function of $r$. For drops with a radius of $4.3 \mathrm{~mm}$, when initially undistorted, and an evaporation rate per unit area at the surface of $\dot{J}=1.6 \times 10^{-7} \mathrm{~g} \mathrm{~cm}^{-2} \mathrm{~s}^{-1}$, the results are as shown in Fig. 3(b). The gap is found to be uniform for small $r$, but found to have a "lip" near the edge of the area of apparent contact. The distance of the closest approach of the drops at the lip is found to be $1 \mu \mathrm{m}$, while at the center the gap is $7 \mu \mathrm{m}$. The separation is thus sufficiently large everywhere that Van der Waals interactions can be neglected. In our experiments we were unable to resolve any space between the drops, but our resolution was limited to about $100 \mu \mathrm{m}$.

There are a number of other hydrodynamic effects which we have considered. These include Marangoni flow arising from spatial variations in the surface tension, natural convection in the liquid, and flow of the liquid induced by the drag exerted by the gas flow. Under the conditions of the present experiment, inclusion of these effects appears to make only minor changes in the results just obtained. A more interesting question concerns the stability of the flow. The above analysis predicts that, as long as $\dot{J}$ is positive, there will always be noncoalescence, i.e., the equations have a solution in which there is a finite gap between the drops. This result is based, however, on the assumption of axial symmetry and does not allow for instabilities in which first contact is made between the drops at a single point around the lip. 
This research is supported by NASA Grant NAGW3359. D. L.W. also acknowledges additional NASA support.

[1] E. H. Brandt, Science 24, 349 (1989).

[2] The molar susceptibility of ${ }^{4} \mathrm{He}$ has been calculated to be $\chi=-1.8914 \times 10^{-6}$. See R. E. Glick, J. Phys. Chem. 65, 1552 (1961).

[3] E. Beaugnon et al., J. Phys. I (France) 3, 399 (1993).

[4] C. G. Paine and G. M. Seidel, Rev. Sci. Instrum. 62, 3022 (1991).
[5] J. G. Leidenfrost, De Aquae Communis Nonnullis Qualitatibus Tractatus (A Tract About Some Qualities of Common Water), Duisburg, 1756; The pertinent portions are reprinted in translation in Int. J. Heat Mass Transf. 9, 1153 (1966).

[6] B. Derjaguin and P. Prokhorov, C. R. Acad. Sci. URSS 54, 507 (1946); reprinted in Prog. Surf. Sci. 43, 273 (1993). The authors observed the noncoalescence of drops of organic liquids in air at room temperature. They concluded that the phenomenon responsible for the formation of a stable gap between the drops involved the flow of an air/organic vapor mixture out of the gap coupled with the counterdiffusion of air inward. These flows were driven by the nonequilibrium conditions of the experiments. 